\title{
Enhanced efficacy of sublingual immunotherapy by liposome-mediated delivery of allergen
}

\author{
This article was published in the following Dove Press journal: \\ International Journal of Nanomedicine \\ Number of times this article has been viewed
}

\author{
Have Aliu ${ }^{1-3}$ \\ Carola Rask' \\ Jens Brimnes' \\ Thomas Lars Andresen ${ }^{2,3}$ \\ 'Immunology Department, In vivo \\ Biology Team, ALK Abelló A/S, \\ Hørsholm, ${ }^{2}$ Department of Micro- \\ and Nanotechnology, Technical \\ University of Denmark, ${ }^{3}$ Center for \\ Nanomedicine and Theranostics, \\ Technical University of Denmark, \\ Kgs Lyngby, Denmark
}

\begin{abstract}
Immunotherapy by sublingual administration of allergens provides high patient compliance and has emerged as an alternative to subcutaneous immunotherapy for the treatment of IgE-associated allergic diseases. However, sublingual immunotherapy (SLIT) can cause adverse events. Development of allergen delivery systems enabling more efficient delivery and hence lower allergen load might reduce the adverse events. In the present study, we have investigated neutral and cationic liposomes as delivery systems of ovalbumin (OVA), as a model allergen, in an OVA-induced allergic airway inflammation model. We investigated the liposome carriers' ability to improve tolerance induction of antigens compared to the corresponding dose of free OVA. Mice were treated sublingually over 2 weeks with free or liposome encapsulated OVA followed by intraperitoneal injections and intranasal challenge. Mice sublingually treated with OVA-liposomes showed a significant reduction of airway eosinophilia and splenocyte proliferation in comparison to free OVA. A similar nonsignificant pattern was seen for OVA-specific IgE antibodies. In addition, reduced levels of interferon- $\gamma$ and interleukin- 5 were observed in spleen cell culture supernatants from OVA-liposome-treated mice compared to the sham-treated group. In conclusion, in vivo efficacy data showed that prophylactic SLIT with OVA-liposomes is significantly more effective in preventing allergic inflammation than the corresponding dose of free OVA.
\end{abstract}

Keywords: sublingual immunotherapy, drug delivery, allergy, liposome

\section{Introduction}

The prevalence of IgE-mediated allergic diseases has increased more than two-fold during the last decades, and currently $>30 \%$ of the population in industrialized countries are allergic to aeroallergens such as pollens and house dust mites. ${ }^{1,2}$ Allergenspecific immunotherapy (AIT) is an effective therapy for IgE-mediated respiratory allergic diseases and has a potentially sustained effect after completed treatment., Sublingual immunotherapy (SLIT), formulated as fluids or as fast dissolving allergen immunotherapy tablets, demonstrates safety and clinical efficacy and has recently emerged as an alternative to subcutaneous immunotherapy (SCIT) with improvements on adverse events. ${ }^{3,5-7}$ The immunological mechanisms of SLIT are not entirely understood; however, it is believed that sublingually administered allergens are taken up by antigen presenting cells (APCs), mainly dendritic cells (DCs), and thereafter processed while the APCs migrate to regional lymph nodes to present the antigen. ${ }^{8,9}$ SLIT has been shown to induce a shift from the allergy promoting Th2 cells (interleukin [IL]-4, IL-5, and IL-13) toward Th1 cells (interferon [IFN]- $\gamma$ ), induce allergen-specific regulatory T-cells, and produce allergen-specific IgA and $\mathrm{IgG}$, mainly IgG4. ${ }^{10-13}$ The treatment duration for SLIT is 3-5 years and even though SLIT is considered to have a better safety profile than SCIT, ${ }^{14}$ it can cause local adverse events and clinical
Correspondence: Thomas Lars Andresen Technical University of Denmark, Department of Micro- and Nanotechnology Building 345B, Room 050, 2800 Kgs Lyngby, Denmark Email thomas.andresen@nanotech.dtu.dk
International Journal of Nanomedicine 2017:12 8377-8388

8377 
supervision is required for the first administration. ${ }^{12,13}$ SLIT relies on high allergen extract doses compared to SCIT ${ }^{11}$ and adverse events can potentially be reduced if the administration dose is decreased which in turn will lead to an improved safety profile.

Encapsulation of allergens in liposomes that can act as allergen delivery systems, ${ }^{15}$ offers the ability to protect the allergen from degradation, potentially aid transport within tissues, and in turn target APCs. ${ }^{16}$ Thus, the use of liposomes as a delivery system could decrease the allergen dose required for successful SLIT and improve the safety profile. Liposomes are able to entrap proteins in the aqueous interior and have been widely used as drug delivery carriers due to their high biocompatibility. ${ }^{17}$ Different parameters such as size, surface charge, composition, and mechanical stability can be controlled by lipid composition, ${ }^{18}$ which may affect transport through sublingual mucosa and immune cell targeting. Nanocarrier based drugs (including liposomes) are under investigation for treatment of a range of diseases, ${ }^{19-21}$ and a number of technologies have reached late stage clinical development, while others have reached the market. ${ }^{22}$ It is the gold standard in nanocarrier technology for in vivo use to surface coat the nanoparticles with polyethylene glycol (PEG), which masks the nanocarrier from the mononuclear phagocytic system and thereby increases nanocarrier half-life in blood after intravenous administration. Interestingly, it has also been shown that PEG can change the adhesion properties between nanoparticles and mucin fibers in mucosal tissues, and as a result may enhance their penetration through layers of sublingual mucosa. ${ }^{23,24}$ As of today, none of the commercially available SLIT vaccines contain any adjuvants. ${ }^{25}$ However, a few studies have shown positive results in murine SLIT models using positively charged maltodextrin and chitosan based microparticles as antigen carriers for mucosal vaccination, where the immune response was enhanced through DC targeting. ${ }^{26,27}$ One study showed that sublingual administration of ovalbumin (OVA) formulated with mucoadhesive maltodextrin matrix was very effective in reducing the airway eosinophil accumulation and decreasing Th2 allergic responses in an OVA-induced allergic airway inflammation model. ${ }^{26}$ Another SLIT study showed that cationic chitosan based nanoparticles carrying OVA could target DC and offer a tendency in lowering Th2 responses when administered sublingually after sensitization in an asthma model. ${ }^{27}$ To our knowledge, liposomes have not yet been investigated for SLIT applications.

In this paper, we have investigated the possibility of developing liposomes as a new allergen delivery system for improving efficacy of SLIT in an OVA-induced allergic airway inflammation model. OVA was encapsulated in liposomes with varying amounts of cationic lipids and was investigated for the ability to improve allergen-specific tolerance compared to free OVA.

\section{Materials and methods Chemicals}

1-palmitoyl-2-oleoyl-sn-glycero-3-phosphocholine (POPC), 1,2-dioleoyl-sn-glycero-3-phosphocholine (DOPC), 1,2dioleoyl-3-trimethylammonium-propane (DOTAP), 1,2-dioleoyl-sn-glycero-3-phosphoethanolamine-N(methoxy[PEG]-1000) (ammonium salt) (DOPE-PEG1000), and 1,2-dioleoyl-sn-glycero-3-phosphoethanolamine-N(methoxy[PEG]-2000) (ammonium salt) (DOPE-PEG2000) were purchased from Avanti Polar Lipids (Alabaster, AL, USA). OVA grade V from chicken egg white (OVA) was purchased from Sigma Aldrich (St Louis, MO, USA) and phosphate-buffered saline (PBS) buffer was obtained from Lonza (Capsugel Bornem, Belgium).

\section{Liposome preparation}

Stock solutions of $100 \mathrm{mM}$ of each lipid used were prepared in chloroform:methanol (9:1) in $8 \mathrm{~mL}$ flat bottom glass vials, and then mixed into a homogeneous mixture at the desired molar ratios. The organic solvent was evaporated in a fume head using dry nitrogen steam for 3 hours. Further, the lipid film was thoroughly dried using a vacuum system to form a thin layer lipid film on the bottom of the vial. The lipid film was hydrated at room temperature in PBS containing the desired amount of OVA $(100 \mathrm{mg} / \mathrm{mL}$ for the in vivo studies). The vials were stirred for 10 seconds every fifth minute during 1 hour and, as a result, $100 \mathrm{mM}$ multilamellar liposomes encapsulating OVA were formed. Liposomes were extruded 21 times through a nuclepore polycarbonate membrane with a pore size of 100 nm from Whatman (Maidstone, UK), using the Avanti-syringe extruder. Liposomes were formulated with a total lipid concentration of $100 \mathrm{mM}$ as mixtures of POPC:DOTAP:DOPEPEG1000 lipids and DOPC:DOTAP:DOPE-PEG1000 lipids. Different molar ratios with the above mixtures were formulated: 98:1:1 (OVA-POPC neutral) and 93:6:1 (OVA-POPC cationic), as well as 98:1:1 (OVA-DOPC neutral) and 93:6:1 (OVA-DOPC cationic). Empty liposomes without OVA were formulated as negative control with the same lipid composition as the OVA-liposomes. Size exclusion chromatography (SEC), using a $20 \mathrm{~cm}$ column packed with Sepharose CL-4b from GE Healthcare Europe GmbH (Freiburg, Germany), was used to separate liposome encapsulated OVA (OVA-liposomes) 
from free OVA. Thereafter, the liposomes were centrifuged in Amicon ultra-15 centrifugal filter units 100,000 MWCO from Merck Millipore (Billerica, MA, USA) to the initial volume prior to SEC (1 mL), to ensure sufficiently high concentration for in vivo use.

\section{Size measurement of liposomes}

The liposome samples were diluted to a final lipid concentration of $0.1 \mathrm{mM}$ for measurement in PBS and the size and polydispersity index (PDI) of the liposomes were measured as replicates $5 \times$ for 30 seconds each using a ZetaPALS dynamic light scattering (DLS) system from Brookhaven Instruments Corporation (Holtsville, NY, USA).

\section{Lipid and encapsulated OVA quantification of the liposomes}

Amino acid analysis (AAA) was used for quantifying soluble or encapsulated OVA prior to SLIT. In short, samples were hydrolyzed for 24 hours in $6 \mathrm{M} \mathrm{HCl}$ where amino acids were separated by ion exchange chromatography. After oxidation and derivatization with ortho-phthalaldehyde, the amino acids were detected using a fluorescence detector (Waters M 474; Waters Corporation, Milford, MA, USA) with $338 \mathrm{~nm}$ bandpass excitation filter and $450 \mathrm{~nm}$ long-pass emission filter. A standard mixture containing $1 \mathrm{nmol}$ of the amino acids was used to identify and quantify each amino acid. ${ }^{28}$ Lipid concentration was determined by measuring the total phosphorous content with inductively coupled plasma mass spectrometry (ICP-MS) using a Dionex ICS-5000+ system (Thermo Fisher Scientific, Waltham, MA, USA). Samples were analyzed by diluting 5,000-fold in $2 \% \mathrm{HCl}$ containing $10 \mathrm{ppb}$ of gallium as an internal standard.

\section{OVA leakage from liposomes}

OVA-POPC cationic and OVA-DOPC cationic liposomes were preserved at $4^{\circ} \mathrm{C}$ in aliquots of $200 \mu \mathrm{L}$, in order to study OVA leakage from the liposomes over time. Leakage was assessed by centrifugation of the liposome samples at $1,000 \times g$ in Amicon ultra- 0.5 centrifugal filter unit with Ultracel-100 membrane 100,000 MWCO filter units from Merck Millipore at $5^{\circ} \mathrm{C}$ for 3 hours. The leakage of OVA was determined by analyzing the filtrate with AAA and comparing to the OVA concentration in the batch.

\section{Animals}

Six-week old Balb/cJ female mice were purchased from Taconic, Ejby, Denmark and maintained on an OVA-free diet. The mice were housed in a pathogen-free environment with 12 hour light, 12 hour dark cycles. All experiments described in this report were conducted in accordance with Danish legislation as stipulated in the Animal Experimentation Act issued by the Ministry of Environment and Food of Denmark. Ethical and legal permissions were obtained prior to the start of the study by the Supervisory Authority on Animal Testing in Glostrup, Denmark.

\section{In vivo experimental design}

Mice were treated sublingually 5 times/week with either $50 \mu \mathrm{g}$ of OVA-liposomes, a corresponding dose of free OVA $(50 \mu \mathrm{g})$, a 10-fold higher dose of free OVA (positive control for treatment effect), or PBS buffer during a 2-week period (Monday-Friday). SLIT was performed by holding mice in the scruff and applying $10 \mu \mathrm{L}$ of the respective treatment under the tongue. Mice were fixed for additional 20 seconds to prevent immediate swallowing of the solution. Thereafter, they were sensitized by two intraperitoneal (i.p) injections of $25 \mu \mathrm{g}$ alum-adsorbed OVA in $250 \mu \mathrm{L}$ buffer solution at day 15 and 29, followed by three intranasal challenges with $10 \mu \mathrm{g}$ OVA in $25 \mu \mathrm{L}$ buffer solution at day 36-38, and were sacrificed at day 39 (Figure 1). Eosinophils in bronchoalveolar lavage (BAL), allergen-specific $\operatorname{IgE}$ in serum, in vitro proliferation, and Th2 cytokine responses

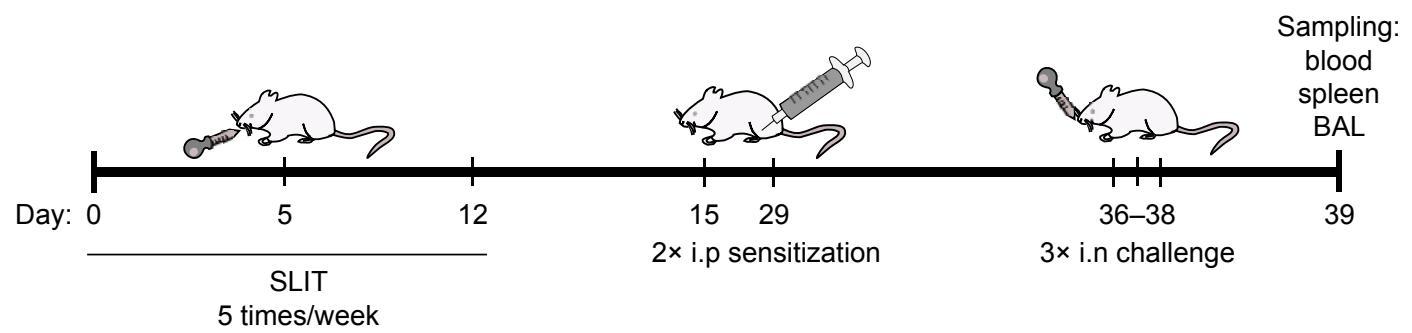

Figure I Schematic image of prophylactic experimental design.

Notes: Mice were treated sublingually 5 times/week with $10 \mu \mathrm{L}$ of OVA-liposomes ( $50 \mu \mathrm{g}$ OVA), free OVA (50 or $500 \mu \mathrm{g})$, or PBS buffer. All mice were subsequently sensitized by two i.p injections with $25 \mu \mathrm{g}$ alum-adsorbed OVA in $250 \mu \mathrm{L}$ buffer solution followed by i.n challenge with $10 \mu \mathrm{g}$ of OVA in $25 \mu \mathrm{L}$ buffer solution over 3 consecutive days. Mice were sacrificed the following day. Blood and spleen were collected and BAL was performed.

Abbreviations: OVA, ovalbumin; PBS, phosphate-buffered saline; i.p, intraperitoneal; BAL, bronchoalveolar lavage; i.n, intranasal; SLIT, sublingual immunotherapy. 
from spleen cells were used as readouts for SLIT efficacy. Control animals were sham-SLIT-treated with PBS buffer as negative control and OVA $500 \mu \mathrm{g} / \mathrm{SLIT}$ dose as positive treatment control. Each experimental group was composed of eight mice.

\section{Eosinophils measurements in BAL}

BAL was performed by using $750 \mu \mathrm{L}$ of Hank's balanced salt solution (Thermo Fisher Scientific) complemented with protease inhibitor cocktail tablet (Roche, Basel, Switzerland). The BAL fluid was centrifuged for 7 minutes at $400 \times g$ at $8^{\circ} \mathrm{C}$ and the pellet, containing the cells, was resuspended in $100 \mu \mathrm{L}$ PBS. Cells were differentially counted by a Sysmex XT1800i hematology analyzer (Sysmex, Kobe, Japan).

\section{OVA IgE quantification of serum}

Serum was prepared by centrifuging blood in BD Microtainer serum separator tubes (BD, Tense, Belgium) for 7 minutes, $400 \times g$ at room temperature. OVA IgE was measured using mouse OVA IgE enzyme-linked immunosorbent assay (ELISA) kit (MD Bioproducts, Zurich, Switzerland). In short, standards and analytical samples were diluted and added to the wells. OVA IgE was detected by adding the biotin conjugate and streptavidin-horseradish peroxidase. The plates were developed with substrate and stopped after 15 minutes. Absorbance was measured with an ELISA reader (ultra microplate reader, EL808; BioTek Instruments, Inc, Winooski, VT, USA) at $450 \mathrm{~nm}$.

\section{Cell proliferation and cytokine production}

The spleen was processed to a single cell suspension by squeezing through a falcon nylon filter (VWR International, Denmark) and washed three times in RPMI-1640 (Lonza) supplemented with $0.05 \mathrm{mg} / \mathrm{mL}$ gentamycin (Thermo Fisher Scientific). Cells were dispersed and counted to a concentration of $3.0 \times 10^{6}$ cells $/ \mathrm{mL}$ in RPMI-1640 medium containing $1 \%$ Nutridoma (Roche), $1.5 \mathrm{mM}$ monothioglycerol (Sigma), $0.05 \mathrm{mg} / \mathrm{mL}$ gentamycin, and $2.5 \%$ fetal calf serum (Thermo Fisher Scientific). In total, $5.4 \times 10^{5}$ and $2.4 \times 10^{5}$ cells were plated on a flat bottomed 96-well Nunc plate for assessment of proliferation and cytokine production. Cells were stimulated with a final concentration of 5,25 , or $125 \mu \mathrm{g} / \mathrm{mL}$ of OVA and the proliferation plates were incubated for 6 days at $37^{\circ} \mathrm{C}$ and $5.5 \% \mathrm{CO}_{2}$. The cells were treated with ${ }^{3} \mathrm{H}$-thymidine for the last 17 hours and splenocyte proliferation was measured by counting the incorporated radiolabel on a Wallac Microbeta 1450 liquid scintillation counter
(Wallac, Wellesley, USA) after harvesting the cells on a Tomtec-96-well plate harvester (Tomtec Inc, Hamden, CT, USA). For measurement of cytokine levels, the cell concentration in the wells was adjusted to $5.4 \times 10^{5}$ and supernatants were collected after 5 days of culture.

\section{Cytokine measurement by ELISA-based method}

The cytokine levels were measured on a custom made MULTI-SPOT $^{\circledR}, 7$ Spot Mouse 6plex from Meso Scale Discovery (Rockville, MD, USA), where IFN- $\gamma$, IL-4, IL-5, IL-10, IL-13, and IL-17 analytes were measured according to their manual. In short, samples and calibrators are added to cytokine pre-coated wells followed by addition of detection antibody. Read buffer was added and the plates were analyzed in the MSD reader (MESO Sector S600) within 15 minutes.

\section{Data analysis}

Statistical analyses were performed with GraphPad Prism software program (version 6.1; GraphPad Software Inc, La Jolla, CA, USA) using one-way analysis of variance or Kruskal-Wallis test and $P$-values were adjusted for multiple comparisons by Holm-Sidak's and Dunn's test, respectively. $P$-values $<0.05$ are considered significant, where $*$ indicates $P<0.05$, ** indicates $P<0.01, * * *$ indicates $P<0.005$, and $* * * *$ indicates $P<0.001$. Data are expressed as mean value of the group + standard error of means.

\section{Results}

\section{Optimization of liposomes}

SLIT treatment in mice generally requires a high concentration of OVA to induce tolerance. It was therefore of interest to encapsulate OVA in the liposome formulations in order to lower the SLIT dose. Different factors affecting the encapsulation capacity were investigated. A main factor that proved to be essential in the liposomal formulation was the incorporation of PEG on the liposome surface, as the extrusion of OVA-liposomes in the absence of PEG proved to be very challenging when using a high concentration of OVA. Liposomes were formulated with either POPC or DOPC as the main lipid components. Different amounts of the cationic lipid DOTAP (net charge of +1 ), were added to the liposome formulations for obtaining liposomes with variable cationic surface charge. OVA was encapsulated by hydrating a lipid film in the presence of OVA. OVA is negatively charged at isotonic $\mathrm{pH}$ with an isoelectric point of 4.8 , and is believed to provoke aggregation complexes with positively charged liposomes by electrostatic interaction. ${ }^{29}$ To circumvent 
aggregation, two different sizes of PEG polymers attached to DOPE lipids, DOPE-PEG1000 and DOPE-PEG2000, were used in different concentrations (Table 1).

Size measurements of OVA-liposomes showed no difference between DOPE-PEG1000 and DOPE-PEG2000. However, increasing the PEG-lipid concentrations resulted in larger OVA-liposomes with high PDI. In addition to the above-mentioned parameters, both ultrasonication (probe and bath) and extrusion were tested for the ability of these methods to obtain homogeneous liposomes in high OVA and lipid concentration. The experimental results showed that extrusion gave more homogeneous liposome populations and smaller average size than ultrasonication $(\sim 150 \mathrm{~nm}$ for extrusion and $\sim 300 \mathrm{~nm}$ for sonication [frequency $50 \mathrm{kHz}$ ] at high concentration of OVA and lipid). In addition, $10 \mathrm{mM}$ acetate buffer, 10 mM 2-(N-Morpholino)ethanesulfonic acid sodium salt (MES) buffer, $10 \mathrm{mM}$ phosphate buffer including $37.5 \mathrm{mM}$ $\mathrm{NaCl}$ with $\mathrm{pH}$ ranging between 4.0 and 7.4 were tested, which did not improve the liposome preparations in terms of easier extrusion (data not shown). After evaluating the formulation procedure, OVA as well as liposomal concentration were increased to $100 \mathrm{mg} / \mathrm{mL}$ and $100 \mathrm{mM}$, respectively, giving the highest degree of encapsulated OVA in the liposomes.

\section{OVA entrapment and lipid concentration of liposomes}

Upon optimization of the liposome preparation procedure, four different OVA-liposome systems were chosen for further studies where size stability was evaluated. The four liposome systems had various degrees of cationic lipid and were based on either POPC or DOPC, and DOPE-PEG1000 as described in Table 2.

SEC was used to separate liposome encapsulated OVA from free OVA after hydrating lipid films in the presence of OVA and after extrusion. The liposomes were further concentrated using centrifuge filters, after which the OVA encapsulation was evaluated for the different formulations. The remaining OVA concentration varied between 6.5 and $7.5 \mathrm{mg} / \mathrm{mL}$, giving an encapsulation efficiency of around 10\% (Table 2). Total lipid concentration of the different liposome formulations was quantified to be between 70 and $85 \mathrm{mM}$, by measuring the phosphorus in the samples using ICP-MS. The liposomes were used immediately after protein quantification in the in vivo studies.

\section{Characterization and physical stability of liposomes}

Size distributions were measured using DLS, which showed that the mean particle diameters were between 140 and
Table I Different parameters tested for optimizing liposome formulations to obtain the highest possible encapsulated OVA concentration for SLIT treatment

\begin{tabular}{|c|c|c|c|c|c|}
\hline $\begin{array}{l}\text { Lipid } \\
\text { composition } \\
\text { (molar ratio) }\end{array}$ & $\begin{array}{l}\text { Theoretical } \\
\text { lipid conc } \\
(\mathrm{mM})\end{array}$ & $\begin{array}{l}\text { Theoretical } \\
\text { OVA conc } \\
(\mathrm{mg} / \mathrm{mL})\end{array}$ & $\begin{array}{l}\text { Lipid to } \\
\text { protein } \\
\text { ratio }\end{array}$ & $\begin{array}{l}\text { Size } \\
(\mathrm{nm})^{*}\end{array}$ & PDI* \\
\hline \multicolumn{6}{|c|}{ POPC:DOTAP:DOPE-PEG2000 } \\
\hline $93: 6: 1$ & 13 & 5 & 2.6 & 170 & 0.11 \\
\hline $88: 11: 1$ & 13 & 5 & 2.6 & 315 & 0.3 \\
\hline $83: 16: 1$ & 13 & 5 & 2.6 & 601 & 0.35 \\
\hline $90: 7.5: 2.5$ & 13 & 5 & 2.6 & 160 & 0.12 \\
\hline $85: 12.5: 2.5$ & 13 & 5 & 2.6 & 310 & 0.29 \\
\hline $80: 17.5: 2.5$ & 13 & 5 & 2.6 & 440 & 0.3 \\
\hline $93: 6: 1$ & 26 & 5 & 5.2 & 143 & 0.16 \\
\hline $90: 7.5: 2.5$ & 26 & 5 & 5.2 & 156 & 0.15 \\
\hline \multicolumn{6}{|c|}{ POPC:DOTAP:DOPE-PEG I000 } \\
\hline $93: 6: 1$ & 13 & 5 & 2.6 & 170 & 0.1 \\
\hline $90: 7.5: 2.5$ & 13 & 5 & 2.6 & 175 & 0.14 \\
\hline $93: 6: 1$ & 26 & 5 & 5.2 & 153 & 0.13 \\
\hline $90: 7.5: 2.5$ & 26 & 5 & 5.2 & 186 & 0.22 \\
\hline $93: 6: 1$ & 50 & 20 & 2.5 & 190 & 0.9 \\
\hline $93: 6: 1$ & 75 & 30 & 2.5 & 184 & 0.08 \\
\hline $93: 6: 1$ & 75 & 50 & 1.5 & 193 & 0.08 \\
\hline $93: 6: 1$ & 75 & 50 & 1.5 & 178 & 0.06 \\
\hline $93: 6: 1$ & 100 & 66.7 & 1.5 & 154 & 0.1 \\
\hline $93: 6: 1$ & 100 & 100 & I & 176 & 0.09 \\
\hline
\end{tabular}

Notes: Lipid concentration, OVA concentration, and ratio are based on theoretical values. *Size and PDI indicate experimental values.

Abbreviations: OVA, ovalbumin; SLIT, sublingual immunotherapy; PDI, polydispersity index; conc, concentration; POPC, I-palmitoyl-2-oleoyl-sn-glycero-3-phosphocholine; DOTAP, I,2-dioleoyl-3-trimethylammonium-propane; DOPE-PEG2000, I,2-dioleoylsn-glycero-3-phosphoethanolamine-N-[methoxy(polyethylene glycol)-2000]; DOPEPEG I000, I,2-dioleoyl-sn-glycero-3-phosphoethanolamine-N-[methoxy(polyethylene glycol)-1000].

$155 \mathrm{~nm}$ with PDI of around 0.1 for both OVA-POPC and OVA-DOPC liposomes. The diameter of "empty" POPC or DOPC liposomes with cationic charge (containing only PBS) was used as a comparison and was in the same size range (Figure 2).

Physical stability tests of samples preserved at $4^{\circ} \mathrm{C}$ over a period of 4.5 months showed that the sizes of the liposomes were stable during the stability testing period (Figure 2) and did not aggregate. Overall, the results demonstrate that the particles are homogeneous and stable for a period of at least 4.5 months. Additional studies of OVA-POPC cationic liposomes showed physical stability for at least 7 months when evaluated monthly by measuring the diameter and PDI (data not shown).

\section{OVA encapsulation stability in liposomes}

To study the leakage over time of OVA from the liposomes, OVA-POPC and OVA-DOPC cationic liposomes preserved at $4^{\circ} \mathrm{C}$ were centrifuged through centrifuge filters at $5^{\circ} \mathrm{C}$ allowing the OVA to pass and retaining the liposome 
Table 2 Characterization of OVA-liposomes for in vivo experiments

\begin{tabular}{|c|c|c|c|c|c|c|}
\hline $\begin{array}{l}\text { Lipid } \\
\text { composition }\end{array}$ & Short name & $\begin{array}{l}\text { Lipid conc } \\
(\mathrm{mM})\end{array}$ & $\begin{array}{l}\text { Diameter } \\
(\mathrm{nm})\end{array}$ & PDI & $\begin{array}{l}\text { OVA encapsulation } \\
(\mathrm{mg} / \mathrm{mL})\end{array}$ & $\begin{array}{l}\text { Lipid to } \\
\text { protein ratio }\end{array}$ \\
\hline \multicolumn{7}{|c|}{ POPC/DOTAP/DOPE-PEGI000 } \\
\hline $98: 1: 1$ & OVA-POPC neutral & 80.9 & 148 & 0.088 & 7.52 & 10.8 \\
\hline 93:6:1 & OVA-POPC cationic & 69.8 & 140 & 0.093 & 7.14 & 9.8 \\
\hline \multicolumn{7}{|c|}{ DOPC/DOTAP/DOPE-PEGI000 } \\
\hline $98: 1: 1$ & OVA-DOPC neutral & 83.9 & $|5|$ & 0.098 & 6.39 & 13.1 \\
\hline $93: 6: 1$ & OVA-DOPC cationic & 71.7 & 153 & 0.094 & 6.76 & 10.6 \\
\hline
\end{tabular}

Abbreviations: OVA, ovalbumin; PDI, polydispersity index; conc, concentration; POPC, I-palmitoyl-2-oleoyl-sn-glycero-3-phosphocholine; DOPC, I,2-dioleoyl-sn-glycero-3phosphocholine; DOTAP, I,2-dioleoyl-3-trimethylammonium-propane; DOPE-PEGI000, I,2-dioleoyl-sn-glycero-3-phosphoethanolamine-N-[methoxy(polyethylene glycol)-1000].

fraction. OVA-POPC cationic liposomes had a concentration of $7.14 \mathrm{mg} / \mathrm{mL}$ OVA immediately after preparation. The protein that passed through the filter in a 1 day stored sample was $2.7 \%$ of the initial concentration (Figure 3 ). OVA concentration in the filtrate increased gradually, reducing the encapsulated OVA to a concentration of $5.2 \mathrm{mg} / \mathrm{mL}$ during the first 2 weeks after which the leakage seemed to be reduced significantly up to the end of the 2 months testing period. We speculate that there might be a certain amount of OVA that is associated with the liposome surface after SEC, which is the fraction that "leaks" (disassociates) from the liposomes over the first weeks. OVA-DOPC cationic liposomes with a start concentration of $6.76 \mathrm{mg} / \mathrm{mL}$ showed a comparable leakage/dissociation of OVA (7.8\% of the encapsulated fraction after the first day). The encapsulated OVA concentration continued to decrease to $5.8 \mathrm{mg} / \mathrm{mL}$ after 2 weeks, after which no further leakage was observed (Figure 3). Again, we therefore speculate that the leakage
A

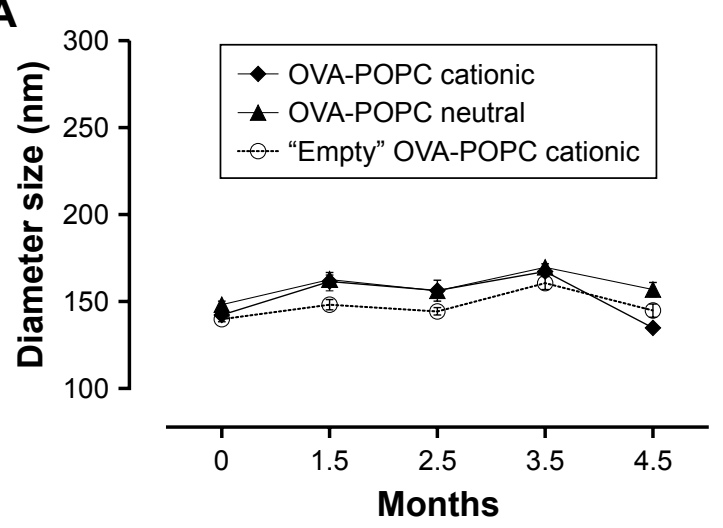

B

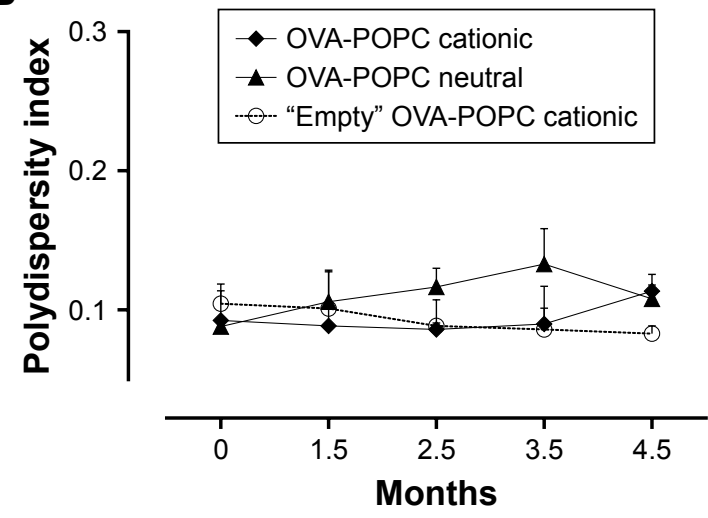

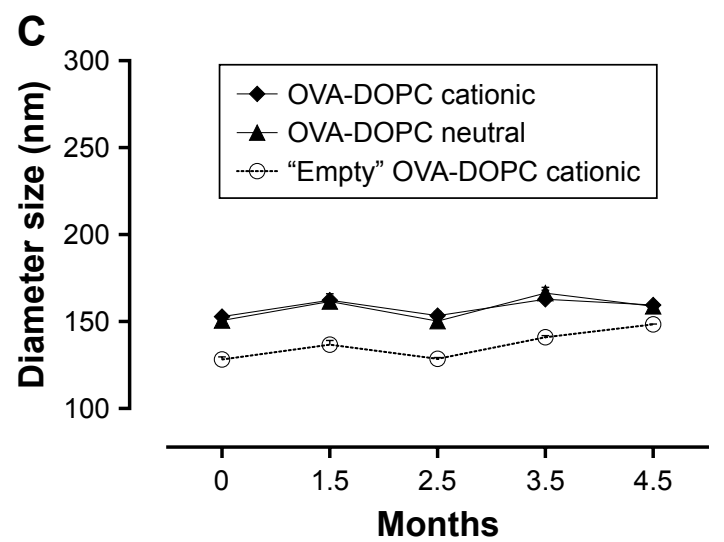

D

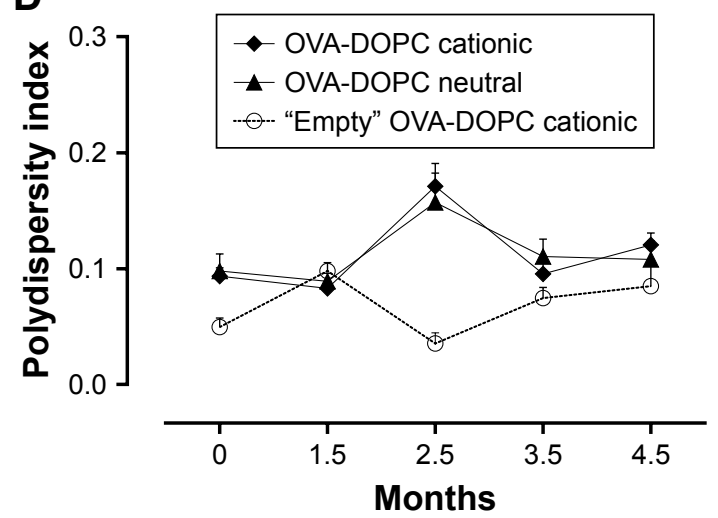

Figure 2 Physical stability and characterization of OVA-liposomes.

Notes: Neutral or cationic formulated OVA-POPC or OVA-DOPC liposomes were tested over a period of 4.5 months using DLS where the liposomes were stored at $4{ }^{\circ} \mathrm{C}$. (A, C) Diameter of OVA-POPC and OVA-DOPC liposomes, respectively, measured as replicates $5 \times$ for 30 seconds each. (B, D) Polydispersity index for OVA-POPC and OVA-DOPC liposomes, respectively. Each time point represents mean \pm SD of two distinct samples.

Abbreviations: OVA, ovalbumin; POPC, I-palmitoyl-2-oleoyl-sn-glycero-3-phosphocholine; DOPC, I,2-dioleoyl-sn-glycero-3-phosphocholine; DLS, dynamic light scattering; SD, standard deviation. 


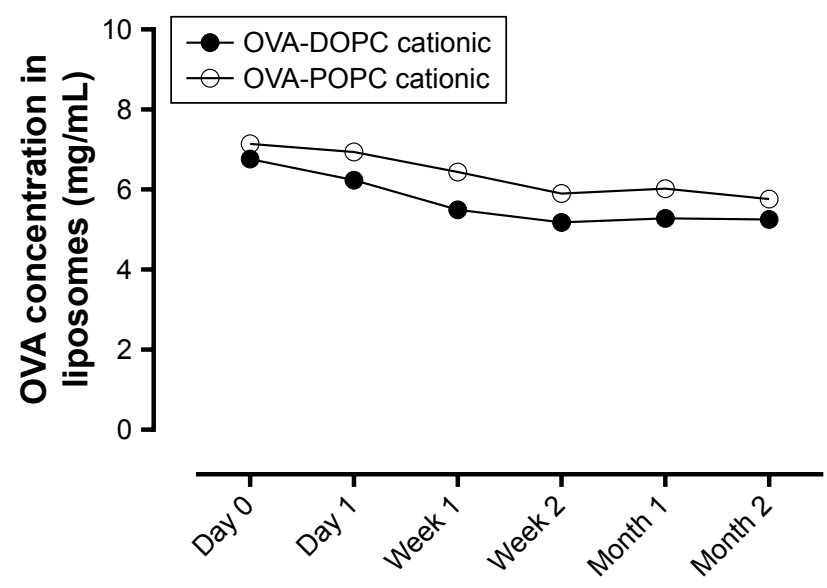

Figure 3 OVA dissociation and leakage from liposomes.

Notes: OVA-liposomes were stored at $4^{\circ} \mathrm{C}$ and centrifuged at different time points using Amicon ultracel-100 membrane 100,000 MWCO filter units. The amount of OVA in the filtrate from both OVA-DOPC and OVA-POPC liposomes was quantified using amino acid analysis.

Abbreviations: OVA, ovalbumin; POPC, I-palmitoyl-2-oleoyl-sn-glycero-3phosphocholine; DOPC, I,2-dioleoyl-sn-glycero-3-phosphocholine.

originates from outer membrane surface-associated OVA that is bound by electrostatic interaction between the cationic membrane and the negatively charged OVA.

\section{Prophylactic SLIT treatment of airway inflammation in mice}

A mouse model of OVA-induced allergic airway inflammation was used to evaluate if prophylactic SLIT with OVA-liposome formulations could reduce the induction of allergy-related inflammation to a higher extent than free OVA. To study the effect of SLIT with OVAliposomes as a function of surface charge and membrane fluidity, we encapsulated OVA in PEGylated neutral and cationic POPC and DOPC liposomes, respectively. As seen in Figure 4A, sublingual treatment with both neutral and cationic OVA-POPC and OVA-DOPC significantly reduced the percentage of eosinophils in BAL fluids compared to the sham-treated group. Moreover, SLIT treatment with neutral OVA-POPC and OVA-DOPC induced a significant reduction in the percentages of eosinophils compared to the SLIT-treated group with a corresponding dose of free OVA. A significant reduction was also observed following SLIT with cationic OVA-DOPC compared to SLIT with free OVA of the same dose. No differences with regard to BAL eosinophils between groups treated with OVA-POPC and OVA-DOPC, respectively, could be observed. SLIT with empty liposomes (containing no OVA only PBS) had no effect on reducing the development of airway inflammation (data not shown).
Allergen-specific IgE was significantly downregulated in mice receiving 2 weeks of SLIT with either neutral OVA-DOPC or cationic OVA-POPC formulations compared to the sham-treated group, whereas SLIT with the corresponding dose of free OVA had no effect on the IgE levels (Figure 4B).

Proliferative responses of splenocytes from mice SLIT treated with OVA-liposomes were decreased two-fold, compared to both the SLIT-treated group with the corresponding dose of free OVA and buffer SLIT-treated group (Figure 4C). Cytokine production was analyzed from the cell supernatant of splenocyte cultures after in vitro stimulation with OVA. Only four mice per group were used for cytokine evaluation which reduced the power of the analysis. Nonetheless, in comparison to sham-treated mice, reduced levels of IFN- $\gamma$ were observed in supernatants of cells generated from mice SLIT treated with both neutral and cationic OVA-POPC and OVA-DOPC liposomes. Levels of IL-5 were downregulated in mice treated with cationic OVA-DOPC liposomes. Cytokine levels in cell supernatants from mice SLIT treated with a corresponding dose of OVA compared to sham-treated mice did not differ. There was a tendency toward decreased levels of both IL-4 and IL-10 in mice SLIT treated with OVA-liposomes. No differences in the levels of IL-13 could be observed between the groups (Figure 5).

\section{Discussion}

IgE-mediated allergic diseases such as allergic asthma and rhinoconjunctivitis can be treated with either AIT or pharmacotherapy. Whereas pharmacotherapy only reduces symptoms, AIT offers the potential for a long-term diseasemodifying effect in addition to symptom relief. ${ }^{6}$ AIT has traditionally been administrated subcutaneously. However, the sublingual route of administration has gained increasing interest since it first appeared in the market in the beginning of the 1990s and was recognized as an alternative to SCIT in children and adults in 2001. ${ }^{30,31}$ The number and severity of systemic side effects for SLIT are much lower compared to SCIT and it has shown significant clinical efficacy compared to placebo. ${ }^{14,32,33}$ It has been demonstrated in many clinical studies $^{34,35}$ and animal models of allergic diseases ${ }^{36,37}$ that the effect of SLIT, both clinical and immunological, depends on the individual allergen dose given with each administration. Concomitantly with an increased efficacy, the number and severity of adverse events also increase with dose. The target profile for an improved SLIT treatment is therefore improved safety with maintained or increased clinical efficacy, and increased patient convenience. We hypothesized that by using 

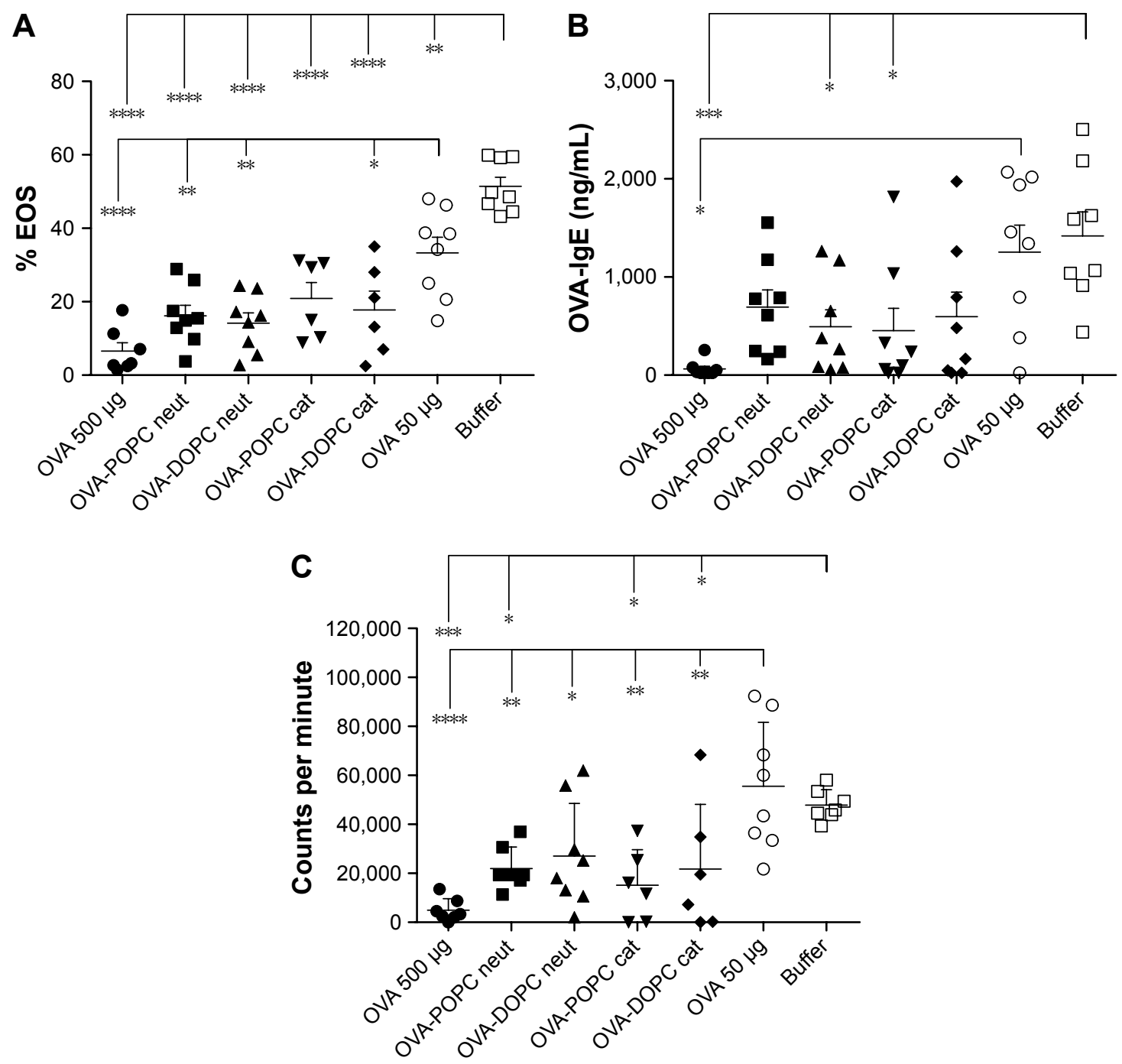

Figure 4 Effect of sublingual immunotherapy on immunological parameters.

Notes: Mice were treated sublingually with $10 \mu \mathrm{L}$ of $50 \mu \mathrm{g}$ OVA either as soluble-free protein or encapsulated in liposomes, $500 \mu \mathrm{g}$ OVA as positive control for treatment, or PBS buffer. They were subsequently sensitized twice by intraperitoneal injections with $25 \mu \mathrm{g}$ of alum-adsorbed OVA in $250 \mu \mathrm{L}$ buffer solution followed by three intranasal challenges of $10 \mu \mathrm{g}$ of OVA in $25 \mu \mathrm{L}$ buffer solution. Mice were sacrificed the following day. (A) The percentages of eosinophils in bronchoalveolar lavage, (B) allergen-specific $\lg$ in serum, and $(\mathbf{C})$ proliferation of splenocytes following 6 days of in vitro stimulation with allergen were analyzed. Each dot represents an individual mouse and the error bars indicate mean + SEM, $n=6-8$. * indicates $P<0.05$, ** indicates $P<0.01$, **** indicates $P<0.005$, and ***** indicates $P<0.001$.

Abbreviations: OVA, ovalbumin; POPC, I-palmitoyl-2-oleoyl-sn-glycero-3-phosphocholine; DOPC, I,2-dioleoyl-sn-glycero-3-phosphocholine; neut, neutral; cat, cationic; EOS, eosinophils; SEM, standard error of means; PBS, phosphate-buffered saline.

liposomes as an allergen carrier, the amount of drug reaching the target site, at any given dose, could be increased compared to SLIT with a soluble-free allergen. Moreover, protection of the allergen inside a liposome may also prevent cross-linking between the allergen and existing allergen-specific IgE and hence contribute to reduced allergen-related side effects.

AIT requires immune cell processing of administered allergen to provide a treatment effect. For SLIT, it is not currently known if the primary effect of allergen processing occurs by APCs locally in the mucus or draining lymph nodes, or if the allergen diffuses to lymph nodes where it is captured and processed by APCs, but the former is the most prevalent hypothesis. ${ }^{8,9}$ Also, it is not well understood which APCs are involved in tolerance induction during SLIT. However, primarily DCs (myeloid and oral Langerhans cells) and macrophages have been described to play a role in antigen presentation after sublingual administration in humans. ${ }^{10,38}$ Likewise, DCs (plasmacytoid, myeloid, and oral Langerhans cells) and macrophage like cells have been suggested in mice. ${ }^{10,39}$ Immature DCs residing in mucosal tissue are highly efficient in probing the tissue environment and internalize nanocarriers such as liposomes by phagocytosis or micropinocytosis. ${ }^{40,41}$ The internalization efficiency is dependent on particle size. In addition, previous reports 

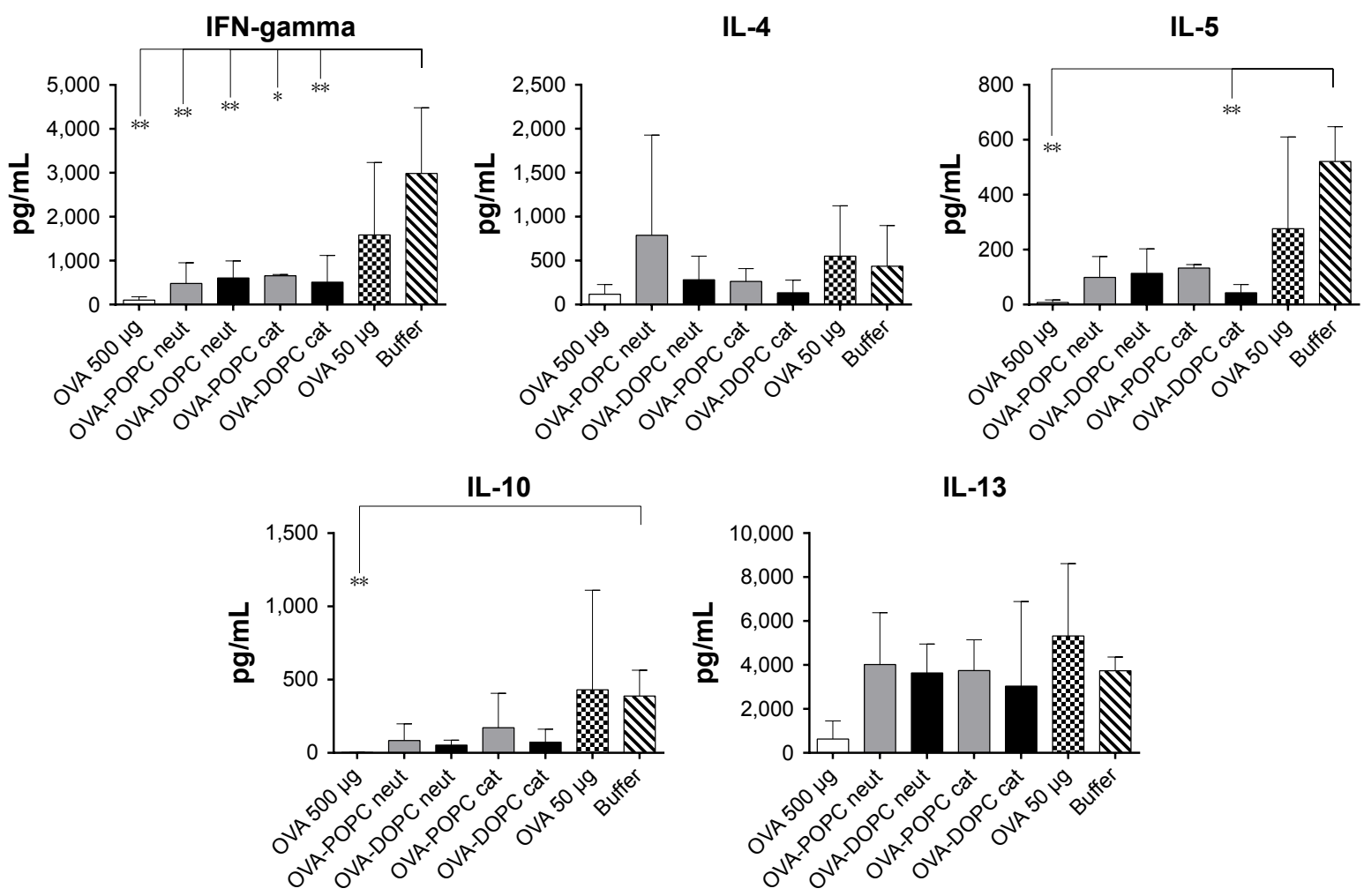

Figure 5 Cytokine levels in supernatants from splenocyte cell cultures of mice treated sublingually with OVA-liposomes, free OVA solution, or PBS buffer.

Notes: Single cell suspensions from spleens were in vitro stimulated during 5 days with $125 \mu \mathrm{g} / \mathrm{mL}$ OVA and cytokine levels were measured using a MULTI-SPOT ${ }^{\circledR}$ Custom Cytokine Assays kit. Error bars indicate mean + SEM, $n=4$. * indicates $P<0.05$, ** indicates $P<0.01$.

Abbreviations: OVA, ovalbumin; POPC, I-palmitoyl-2-oleoyl-sn-glycero-3-phosphocholine; DOPC, I,2-dioleoyl-sn-glycero-3-phosphocholine; neut, neutral; cat, cationic; SEM, standard error of means; PBS, phosphate-buffered saline.

with intracutaneous application indicate that liposomes are capable of migrating from the administered site to the lymph nodes, depending on their size, where smaller liposomes $(<200 \mathrm{~nm})$ drain more efficiently to lymph nodes than larger particles. ${ }^{40,42}$

Allergic and acute inflammatory reactions are rarely seen in oral mucosa which maintains immune homeostasis by tolerating harmless commensals while at the same time recognizes and eliminates pathogens,${ }^{10}$ which makes SLIT an attractive strategy as a site of administration of allergens during AIT. A general challenge with allergens used in SLIT is that they are rapidly cleared from the oral mucosa giving a short period of potential interactions with APCs. One possible approach to circumvent the rapid antigen clearance in the mucosa is to use liposomes as an allergen delivery system to modify clearance characteristics. PEG is a nontoxic polymer that is a gold standard in liposomology to control liposome interactions in biological environments, and PEG has been found to have bioadhesive properties toward mucus layers in tissues. ${ }^{23,43,44}$ Therefore, in order to protect the allergen from rapid clearance and to enhance its penetration through layers of the sublingual mucosa in an effective way, OVA was encapsulated into PEGylated phospholipid liposomes and the membrane surface charge was furthermore investigated as an additional parameter to retain the allergen in the peripheral tissues and increase APC uptake after sublingual administration in mice.

POPC or DOPC lipids were used as the main lipid components in the liposomes used in this study to encapsulate OVA delivery and we investigated the effect of liposome membrane fluidity/rigidity and surface charge for improving prophylactic SLIT in mice with OVA-liposomes in comparison with free OVA. The encapsulation of OVA in the investigated liposomes proved to be a challenge because of the high concentrations of lipid and OVA needed, which was primarily due to the electrostatic interaction between the negatively charged OVA and the positively charged liposome membrane that made extrusion and sonication challenging. It should be noted that this interaction also has a beneficial effect on OVA encapsulation efficiency. Introduction of PEG substantially reduced this challenge and it was possible to prepare liposomes in the desired size range $(<200 \mathrm{~nm})$ and with good stability. The formulated OVA-POPC and OVADOPC liposomes were physically stable for $>4$ months 
when preserved at $4^{\circ} \mathrm{C}$, and the liposomes did not aggregate during this time.

The OVA-POPC and OVA-DOPC liposomes showed a statistically significant reduction of splenocyte proliferation and airway eosinophilia as well as nonsignificant downregulations for OVA-specific IgE antibodies compared to corresponding doses of free OVA. This indicates that prophylactic SLIT with OVA-POPC and OVA-DOPC liposomes prevents induction of allergic parameters when, SLIT is administered prior to allergic sensitization, more efficiently than SLIT treatment with free OVA. The enhanced effect may depend on the electrostatic interactions between cationic liposomes and negatively charged cell membranes, which in turn could improve the uptake and delivery of liposomes by DCs. ${ }^{45}$ Analysis of cytokine production from the spleen revealed a significant downregulation for IFN- $\gamma$ for all liposome formulations compared to the sham-treated group. There was furthermore a tendency for lower levels of IL-5, IL-4, and IL-10 compared to sham-treated mice and free OVA for the cationic liposomes, although these were not significant. The free OVA data (positive control for treatment effect) are in line with previous data from our group and others, ${ }^{26}$ which has shown that SLIT with free OVA can prevent induction of Th2 responses such as airway eosinophilia, allergen-specific IgE, systemic T-cell proliferation, and Th2 cytokines, when SLIT is performed prior to allergic sensitization. ${ }^{4,46}$ The data in the present study indicate that liposomes are capable of improving the most important allergy-associated mechanisms such as cell proliferation and proinflammatory cytokine profiles, except for IL-13, which is an important cytokine in relation to airway inflammation and a major factor in Th2 inflammation and tissue remodeling.

In the present study, the various liposomes tested gave comparable results and all were better than free OVA in inducing a tolerance effect. Future studies could include ultra-deformable liposomes where inspiration can be drawn from the pioneering work of Cevc et al for delivery of drugs across skin. ${ }^{21,47}$ Another parameter that might be considered in the future is to prepare smaller particles that could penetrate easier through mucin fibers in the mucosa. Based on the general literature knowledge, cationic liposomes should result in a better efficacy due to electrostatic interactions with APC, if they translocate. ${ }^{45}$

Safety issues have not been specifically addressed in this study; however, lipids are generally biocompatible and a major constituent of cell membranes. Liposomes have been used in human clinical trials for allergen vaccination using subcutaneous injection where Dermatophagoides pteronyssinus extract encapsulated in DOPC/cholesterol liposomes has been shown to be well tolerated, safe, and efficacious. ${ }^{48}$ However, in the present study we did use a low molar ratio ( $1 \mathrm{~mol} \%$ and $6 \mathrm{~mol} \%$ ) of cationic lipids (DOTAP) to formulate OVA. Cationic lipids have been found to induce in vivo toxicity when used in large amounts. However, we do not expect that the small amounts used in the present study will induce toxicity. Even though we did not observe any adverse effects using the formulations in the present study, more detailed studies of toxicity should be carried out in future investigations.

In conclusion, the OVA-liposomes investigated in this project have a long shelf-life and both OVA-POPC and OVADOPC liposomes used as drug delivery systems in this study enhanced the treatment efficacy when administered sublingually compared to free OVA and consequently prohibit induction of allergic parameters when SLIT is performed prior to allergic sensitization.

\section{Acknowledgments}

We thank Tobias Naesborg, Eva Diemar, Stina Thorup, Sandra Urioste, and Stephanie La Fontaine Svenstrup for their support in the animal facility and in the laboratory. This project was funded by The Danish Agency for Science, Technology and Innovation and ALK Abelló A/S and was carried out at the Technical University of Denmark and at ALK Abelló A/S, Denmark.

\section{Disclosure}

The authors report no conflicts of interest in this work.

\section{References}

1. Pfaar O, Cazan D, Klimek L, Larenas-Linnemann D, Calderon M. Adjuvants for immunotherapy. Curr Opin Allergy Clin Immunol. 2012;12(6):648-657.

2. Upton MN, Mcconnachie A, Mcsharry C, Hart CL, Smith GD, Gillis CR. Intergenerational 20 year trends in the prevalence of asthma and hay fever in adults: the Midspan family study surveys of parents and offspring. BMJ. 2000;321:88-92.

3. Durham SR. Sustained effects of grass pollen AIT. Allergy. 2011; 66(Suppl 95):50-52.

4. Moingeon $\mathrm{P}$, Mascarell L. Induction of tolerance via the sublingual route: mechanisms and applications. Clin Dev Immunol. 2012;2012: 623474.

5. Canonica GW, Passalacqua G. Noninjection routes for immunotherapy. J Allergy Clin Immunol. 2003;111(3):437-448.

6. Durham SR, Emminger W, Kapp A, et al. SQ-standardized sublingual grass immunotherapy: confirmation of disease modification 2 years after 3 years of treatment in a randomized trial. J Allergy Clin Immunol. 2012; 129(3):717-725.

7. Dahl R, Kapp A, Colombo G, et al. Efficacy and safety of sublingual immunotherapy with grass allergen tablets for seasonal allergic rhinoconjunctivitis. J Allergy Clin Immunol. 2006;118(2):434-440.

8. Van Wilsem EJ, Van Hoogstraten IM, Brevé J, Scheper RJ, Kraal G. Dendritic cells of the oral mucosa and the induction of oral tolerance. A local affair. Immunology. 1994;83(1):128-132. 
9. Eriksson K, Ahlfors E, George-Chandy A, Kaiserlian D, Czerkinsky C. Antigen presentation in the murine oral epithelium. Immunology. 1996; 88(1):147-152.

10. Allam J-P, Novak N. Local immunological mechanisms of sublingual immunotherapy. Curr Opin Allergy Clin Immunol. 2011;11(6): 571-578.

11. Moingeon P, Batard T, Fadel R, Frati F, Sieber J, Van Overtvelt L. Immune mechanisms of allergen-specific sublingual immunotherapy. Allergy. 2006;61(2):151-165.

12. Radulovic S, Jacobson MR, Durham SR, Nouri-Aria KT. Grass pollen immunotherapy induces Foxp3-expressing CD4+ CD25+ cells in the nasal mucosa. J Allergy Clin Immunol. 2008;121(6):1467-1472.

13. Scadding GW, Shamji MH, Jacobson MR, et al. Sublingual grass pollen immunotherapy is associated with increases in sublingual Foxp3expressing cells and elevated allergen-specific immunoglobulin G4, immunoglobulin A and serum inhibitory activity for immunoglobulin E-facilitated allergen binding to B. Clin Exp Allergy. 2010;40(4): 598-606.

14. Khinchi MS, Poulsen LK, Carat F, Hansen AB. Clinical efficacy of sublingual and subcutaneous birch pollen allergen-specific immunotherapy: a randomized, placebo-controlled, double-blind, double-dummy study. Allergy. 2004;59:45-53.

15. De Souza Rebouças J, Esparza I, Ferrer M, Sanz ML, Irache JM, Gamazo C. Nanoparticulate adjuvants and delivery systems for allergen immunotherapy. J Biomed Biotechnol. 2012;2012(474605):13.

16. Klippstein R, Pozo D. Nanotechnology-based manipulation of dendritic cells for enhanced immunotherapy strategies. Nanomedicine. 2010;6(4): 523-529.

17. Gregoriadis G, McCormack B, Obrenovic M, Saffie R, Zadi B, Perrie Y. Vaccine entrapment in liposomes. Methods. 1999;19(1):156-162.

18. Torchilin VP. Liposomes as delivery agents for medical imaging. Mol Med Today. 1996;2(6):242-249.

19. Boulaiz H, Alvarez PJ, Ramirez A, et al. Nanomedicine: application areas and development prospects. Int J Mol Sci. 2011;12(5):3303-3321.

20. Li W, Szoka FC. Lipid-based nanoparticles for nucleic acid delivery. Pharm Res. 2007;24(3):438-449.

21. Cevc G, Blume G. Hydrocortisone and dexamethasone in very deformable drug carriers have increased biological potency, prolonged effect, and reduced therapeutic dosage. Biochim Biophys Acta. 2004 1663(1-2):61-73.

22. Chang H-I, Yeh M-K. Clinical development of liposome-based drugs: formulation, characterization, and therapeutic efficacy. Int JNanomedicine. 2012;7:49-60.

23. Lai SK, Wang Y-Y, Hanes J. Mucus-penetrating nanoparticles for drug and gene delivery to mucosal tissues. Adv Drug Deliv Rev. 2009;61(2): $158-171$

24. Gamazo C, Gastaminza G, Ferrer M, Sanz ML, Irache JM. Nanoparticle based-immunotherapy against allergy. Immunotherapy. 2014;6(7): 885-897.

25. Canonica GW, Bousquet J, Casale T, et al. Sub-lingual immunotherapy: World Allergy Organization Position Paper 2009. 2009;64:1-59.

26. Razafindratsita A, Saint-Lu N, Mascarell L, et al. Improvement of sublingual immunotherapy efficacy with a mucoadhesive allergen formulation. J Allergy Clin Immunol. 2007;120(2):278-285.

27. Saint-Lu N, Tourdot S, Razafindratsita A, et al. Targeting the allergen to oral dendritic cells with mucoadhesive chitosan particles enhances tolerance induction. Allergy. 2009;64(7):1003-1013.

28. Barkholt V, Jensen AL. Amino acid analysis: determination of cysteine plus half-cystine in proteins after hydrochlorid acid hydrolosis with a disulfide compound as additive. Anal Biochem. 1989;177:318-322.

29. Xia Y, Sun J, Liang D. Aggregation, fusion, and leakage of liposomes induced by peptides. Langmuir. 2014;30(25):7334-7342.

30. Wilson DR, Lima MT, Durham SR. Sublingual immunotherapy for allergic rhinitis: systematic review and meta-analysis. Allergy. 2005; 60(1):4-12.
31. Penagos M, Compalati E, Tarantini F, et al. Efficacy of sublingual immunotherapy in the treatment of allergic rhinitis in pediatric patients 3 to 18 years of age: a meta-analysis of randomized, placebo-controlled, doubleblind trials. Ann Allergy Asthma Immunol. 2006;97(2):141-148.

32. Mungan D, Misirligil Z, Gürbüz L. Comparison of the efficacy of subcutaneous and sublingual immunotherapy in mite-sensitive patients with rhinitis and asthma - a placebo controlled study. Ann Allergy Asthma Immunol. 1999;82(5):485-490.

33. Saporta D. Efficacy of sublingual immunotherapy versus subcutaneous injection immunotherapy in allergic patients. $J$ Environ Public Health. 2012;2012:492405.

34. Durham SR, Yang WH, Pedersen MR, Johansen N, Rak S. Sublingual immunotherapy with once-daily grass allergen tablets: a randomized controlled trial in seasonal allergic rhinoconjunctivitis. $J$ Allergy Clin Immunol. 2006;117:802-809.

35. Marcucci F, Sensi L, Di Cara G, Incorvaia C, Frati F. Dose dependence of immunological response to sublingual immunotherapy. Allergy. 2005; 60:952-956.

36. Kildsgaard J, Brimnes J, Jacobi H, Lund K. Sublingual immunotherapy in sensitized mice. Ann Allergy Asthma Immunol. 2007;98(4): 366-372.

37. Brimnes J, Kildsgaard J, Jacobi H, Lund K. Sublingual immunotherapy reduces allergic symptoms in a mouse model of rhinitis. Clin Exp Allergy. 2007;37(4):488-497.

38. Mascarell L, Rak S, Worm M, et al. Characterization of oral immune cells in birch pollen-allergic patients: impact of the oral allergy syndrome and sublingual allergen immunotherapy on antigen presenting cells. Allergy. 2015;70(4):408-419.

39. Mascarell L, Saint-Lu N, Moussu H, et al. Oral macrophage-like cells play a key role in tolerance induction following sublingual immunotherapy of asthmatic mice. Mucosal Immunol. 2011;4(6):638-647.

40. Reddy ST, Swartz MA, Hubbell JA. Targeting dendritic cells with biomaterials: developing the next generation of vaccines. Trends Immunol. 2006;27(12):573-579.

41. Kwon YJ, James E, Shastri N, Fre JMJ. In vivo targeting of dendritic cells for activation of cellular immunity using vaccine carriers based on pH-responsive microparticles. Proc Natl Acad Sci U SA. 2005;102(51): 18264-18268.

42. Manolova V, Flace A, Bauer M, Schwarz K, Saudan P, Bachmann MF. Nanoparticles target distinct dendritic cell populations according to their size. Eur J Immunol. 2008;38(5):1404-1413.

43. Yoncheva K, Gómez S, Campanero MA, Gamazo C, Irache JM. Bioadhesive properties of pegylated nanoparticles. Expert Opin Drug Deliv. 2005;2(2):205-218.

44. Lai SK, O'Hanlon DE, Harrold S, et al. Rapid transport of large polymeric nanoparticles in fresh undiluted human mucus. Proc Natl Acad Sci U S A. 2007;104(5):1482-1487.

45. Lonez C, Vandenbranden M, Ruysschaert J-M. Cationic liposomal lipids: from gene carriers to cell signaling. Prog Lipid Res. 2008;47(5): 340-347.

46. Yamada $\mathrm{T}$, Tongu $\mathrm{M}$, Goda $\mathrm{K}$, et al. Sublingual immunotherapy induces regulatory function of IL-10-expressing CD4(+)CD25(+)Foxp3(+) $\mathrm{T}$ cells of cervical lymph nodes in murine allergic rhinitis model. J Allergy. 2012;(490905):1-11.

47. Cevc G, Vierl U. Nanotechnology and the transdermal route: a state of the art review and critical appraisal. $J$ Control Release. 2010;141(3): 277-299.

48. Basomba A, Tabar AI, de Rojas DHF, et al. Allergen vaccination with a liposome-encapsulated extract of Dermatophagoides pteronyssinus: a randomized, double-blind, placebo-controlled trial in asthmatic patients. J Allergy Clin Immunol. 2002;109(6):943-948. 


\section{Publish your work in this journal}

The International Journal of Nanomedicine is an international, peerreviewed journal focusing on the application of nanotechnology in diagnostics, therapeutics, and drug delivery systems throughout the biomedical field. This journal is indexed on PubMed Central, MedLine, CAS, SciSearch $\AA$, Current Contents $\AA /$ Clinical Medicine,

Journal Citation Reports/Science Edition, EMBase, Scopus and the Elsevier Bibliographic databases. The manuscript management system is completely online and includes a very quick and fair peer-review system, which is all easy to use. Visit http://www.dovepress.com/ testimonials.php to read real quotes from published authors.

Submit your manuscript here: http://www.dovepress.com/international-journal-of-nanomedicine-journal 